\title{
Effects of an acute stressor on snack intake in a laboratory environment
}

\author{
N. G. Maloney ${ }^{1}$, K. Giles ${ }^{1}$, C. L. Fyfe ${ }^{1}$, C. Cooper ${ }^{2}$, A. Lorenzo-Arribas ${ }^{3}$, D. B. O'Connor ${ }^{4}$ \\ and A. M. Johnstone ${ }^{1}$ \\ ${ }^{1}$ Rowett Institute of Nutrition and Health, University of Aberdeen, AB21 9SB, ${ }^{2}$ Health Science Building, University of \\ Aberdeen, Foresterhill, Aberdeen AB25 2ZD, ${ }^{3}$ Biomathematics and Statistics Scotland, Rowett Institute, Aberdeen, \\ AB21 $9 S B$ and ${ }^{4}$ Institute of Psychological Sciences, University of Leeds, Leeds, West Yorkshire LS2 9JT
}

Stress can influence our health, directly or indirectly through behavioural change. This includes feeding behaviour, specifically the type and quantity of food consumed ${ }^{(1)}$, where some people will eat more and some people will consume less calories under stress. Longitudinal studies have suggested that elevated stress levels can encourage an increased desire for hedonically pleasing, highly palatable energy dense foods, resulting in an energy intake surplus and possible weight gain. However, there is some controversy as to whether laboratory-based stressors can effectively induce changes in food intake ${ }^{(2)}$. The aim of the present study was to assess whether an acute stressor was effective in capturing a change in eating behaviour in a controlled laboratory setting.

Sixty healthy men and women (mean BMI $25 \cdot 1 \mathrm{~kg} / \mathrm{m}^{2}$ and age 41 years) attended the Human Nutrition Unit on two occasions, relating to 'control' and 'stress' test days. They participated in a standard laboratory stressor adapted from the Trier Social Stress Test (TSST) ${ }^{(3)}$. Saliva samples to monitor cortisol reactivity and recovery to estimate hypothalamic-pituitary-adrenal (HPA) axis response were collected. Participants completed half-hourly Visual Analogue Scales (VAS) measuring subjective ratings of stress and motivation to eat. Assessment of food choice was measured 60 minutes after the stress test using an ad libitum buffet with 24 ready to eat sweet and savoury foods. Volunteers were instructed to eat until comfortably full. Food items were weighed to determine food, energy and nutrient intake from the buffet.

Thirty-two volunteers (mean BMI $25.7 \mathrm{~kg} / \mathrm{m}^{2}$ ) were classified as stress susceptible increase (SSI). They had significantly higher mean energy intake (SEM) on the TSST day compared to their control day (703.4 (12.52) kcal and 438.2 (8.73) kcal, $p=0.003)$. Twenty-six volunteers (mean BMI $24.6 \mathrm{~kg} / \mathrm{m}^{2}$ ) were categorised as stress susceptible decrease (SSD), consuming significantly less on the TSST day compared to their control $(397.4(8.9) \mathrm{kcal}$ and $603.8(10.01) \mathrm{kcal}, p=0.004)$. Two volunteers (mean BMI $\left.22.2 \mathrm{~kg} / \mathrm{m}^{2}\right) \mathrm{were}$ classified as stress resistant (SR), their eating behaviour remained unchanged (154.9 (6.54) kcal and 154.9 (6.54) kcal). There was a statistically significant difference between phenotype group SSD and SSI $(p=0.023)$ for calorie intake. Both SSI and SSD volunteers perceived themselves to be significantly more stressed on TSST day $(p<0 \cdot 001)$, with SSI having a higher level compared to SSD volunteers. Phenotypes SSI and SSD had a significant difference in their hunger levels following the TSST and control with a significant rise 30 minutes after $(p<0 \cdot 001)$. SSD phenotypes also exhibited a significant increase in mean cortisol levels immediately after the TSST, $(5.86 \mathrm{nmol} / \mathrm{L})$ compared to the control $(4.65 \mathrm{nmol} / \mathrm{L})(p=0.004)$ while there was no significant difference in cortisol levels for SSI phenotypes $(p=0 \cdot 258)$.

In conclusion, the laboratory setting proved effective in monitoring individual response following exposure to an artificial stressor to detect changes in eating behaviour and salivary cortisol response. Both physiological and psychological effects appear to influence food intake after acute stress.

The work is funded by the European Union Seventh Framework Programme (FP7/2007-2013) under grant agreement n² 245009 for the 'NeuroFAST' grant.

1. O'Connor DB, Jones J, Conner M et al. (2008) Health Psychol 27, S20-S30.

2. Oliver G, Wardle J, Gibson L, (2000) Psychosom Med 62, pp. 853-865.

3. Newman E, O'Connor DB, Conner M, (2007) Psychoneuroendocrinogy 32, 125-32. 